\title{
Measurement of the Generalized Polarizabilities of the Proton in Virtual Compton Scattering at $Q^{2}=0.92$ and $1.76 \mathrm{GeV}^{2}$ : I. Low Energy Expansion Analysis
}

S. Jaminion, ${ }^{1}$ N. Degrande,${ }^{2}$ G. Laveissière,${ }^{1}$ C. Jutier,${ }^{1,3}$ L. Todor,${ }^{3}$ R. Di Salvo,${ }^{1}$ L. Van Hoorebeke, ${ }^{2}$ L.C. Alexa,${ }^{4}$ B.D. Anderson, ${ }^{5}$ K.A. Aniol, ${ }^{6}$ K. Arundell, ${ }^{7}$ G. Audit,${ }^{8}$ L. Auerbach,${ }^{9}$ F.T. Baker, ${ }^{10}$ M. Baylac, ${ }^{8}$ J. Berthot, ${ }^{1}$ P.Y. Bertin, ${ }^{1}$ W. Bertozzi, ${ }^{11}$ L. Bimbot,${ }^{12}$ W.U. Boeglin, ${ }^{13}$ E.J. Brash, ${ }^{4}$ V. Breton, ${ }^{1}$ H. Breuer, ${ }^{14}$ E. Burtin, ${ }^{8}$ J.R. Calarco, ${ }^{15}$ L.S. Cardman, ${ }^{16}$ C. Cavata, ${ }^{8}$ C.-C. Chang, ${ }^{14}$ J.-P. Chen, ${ }^{16}$ E. Chudakov, ${ }^{16}$ E. Cisbani, ${ }^{17}$ D.S. Dale, ${ }^{18}$ C.W. de Jager, ${ }^{16}$ R. De Leo, ${ }^{19}$ A. Deur,,${ }^{16}$ N. d'Hose, ${ }^{8}$ G.E. Dodge, ${ }^{3}$ J.J. Domingo, ${ }^{16}$ L. Elouadrhiri, ${ }^{16}$ M.B. Epstein, ${ }^{6}$ L.A. Ewell,,${ }^{14}$ J.M. Finn, ${ }^{7}$ K.G. Fissum,${ }^{11}$ H. Fonvieille, ${ }^{1}$ G. Fournier,${ }^{8}$ B. Frois,${ }^{8}$ S. Frullani, ${ }^{17}$ C. Furget, ${ }^{20}$ H. Gao $,{ }^{11}, 21$ J. Gao,${ }^{11}$ F. Garibaldi, ${ }^{17}$ A. Gasparian, ${ }^{22,18}$ S. Gilad, ${ }^{11}$ R. Gilman, ${ }^{23,16}$ A. Glamazdin, ${ }^{24}$ C. Glashausser, ${ }^{23} \mathrm{~J}$. Gomez, ${ }^{16}$ V. Gorbenko, ${ }^{24}$ P. Grenier, ${ }^{1}$ P.A.M. Guichon, ${ }^{8}$ J.O. Hansen, ${ }^{16}$ R. Holmes ${ }^{25}$ M. Holtrop, ${ }^{15}$ C. Howell, ${ }^{21}$ G.M. Huber, ${ }^{4}$ C.E. Hyde-Wright,${ }^{3}$ S. Incerti, ${ }^{9}$ M. Iodice, ${ }^{17}$ J. Jardillier, ${ }^{8}$ M.K. Jones, ${ }^{7},{ }^{16}$ W. Kahl,${ }^{25}$ S. Kato, ${ }^{26}$ A.T. Katramatou, ${ }^{5}$ J.J. Kelly, ${ }^{14}$ S. Kerhoas, ${ }^{8}$ A. Ketikyan, ${ }^{27}$ M. Khayat, ${ }^{5}$ K. Kino, ${ }^{28}$ S. Kox,${ }^{20}$ L.H. Kramer, ${ }^{13}$ K.S. Kumar, ${ }^{29}$ G. Kumbartzki, ${ }^{23}$ M. Kuss, ${ }^{16}$ A. Leone, ${ }^{30}$ J.J. LeRose, ${ }^{16}$ M. Liang ${ }^{16}$ R.A. Lindgren,${ }^{31}$ N. Liyanage, ${ }^{11,31}$ G.J. Lolos,${ }^{4}$ R.W. Lourie,${ }^{32}$ R. Madey, ${ }^{5}$ K. Maeda, ${ }^{28}$ S. Malov, ${ }^{23}$ D.M. Manley ${ }^{5}$ C. Marchand ${ }^{8}$ D. Marchand, ${ }^{8}$ D.J. Margaziotis, ${ }^{6}$ P. Markowitz, ${ }^{13}$ J. Marroncle, ${ }^{8}$ J. Martino, ${ }^{8}$ C.J. Martoff, ${ }^{9}$ K. McCormick, ${ }^{3,23}$ J. McIntyre, ${ }^{23}$ S. Mehrabyan, ${ }^{27}$ F. Merchez,${ }^{20}$ Z.E. Meziani, ${ }^{9}$ R. Michaels,${ }^{16}$ G.W. Miller, ${ }^{29}$ J.Y. Mougey ${ }^{20}$ S.K. Nanda ${ }^{16}$ D. Neyret, ${ }^{8}$ E.A.J.M. Offermann, ${ }^{16}$ Z. Papandreou, ${ }^{4}$ C.F. Perdrisat,${ }^{7}$ R. Perrino, ${ }^{30}$ G.G. Petratos,${ }^{5}$ S. Platchkov ${ }^{8}$ R. Pomatsalyuk,${ }^{24}$ D.L. Prout,${ }^{5}$ V.A. Punjabi, ${ }^{33}$ T. Pussieux, ${ }^{8}$ G. Quémenér, ${ }^{7,20}$ R.D. Ransome,${ }^{23}$ O. Ravel, ${ }^{1}$ J.S. Real,${ }^{20}$ F. Renard,${ }^{8}$ Y. Roblin, ${ }^{1,16}$ D. Rowntree, ${ }^{11}$ G. Rutledge,${ }^{7}$ P.M. Rutt,${ }^{23}$ A. Saha,${ }^{16}$ T. Saito,${ }^{28}$ A.J. Sarty ${ }^{34}$ A. Serdarevic,${ }^{4,}{ }^{16}$ T. Smith, ${ }^{15}$ G. Smirnov ${ }^{1}$ K. Soldi, ${ }^{35}$ P. Sorokin, ${ }^{24}$ P.A. Souder ${ }^{25}$ R. Suleiman, ${ }^{5,11}$ J.A. Templon, ${ }^{10}$ T. Terasawa, ${ }^{28}$ R. Tieulent,${ }^{20}$

E. Tomasi-Gustaffson, ${ }^{8}$ H. Tsubota, ${ }^{28}$ H. Ueno, ${ }^{26}$ P.E. Ulmer,${ }^{3}$ G.M. Urciuoli, ${ }^{17}$ R. Van De Vyver,${ }^{2}$ R.L.J. Van der Meer, ${ }^{4,16}$ P. Vernin, ${ }^{8}$ B. Vlahovic, ${ }^{35}$ H. Voskanyan, ${ }^{27}$ E. Voutier ${ }^{20}$ J.W. Watson, ${ }^{5}$ L.B. Weinstein,${ }^{3}$

K. Wijesooriya, ${ }^{7}$ R. Wilson, ${ }^{36}$ B.B. Wojtsekhowski, ${ }^{16}$ D.G. Zainea, ${ }^{4}$ W-M. Zhang, ${ }^{5}$ J. Zhao, ${ }^{11}$ and Z.-L. Zhou ${ }^{11}$

(The Jefferson Lab Hall A Collaboration)

${ }^{1}$ Université Blaise Pascal/IN2P3, F-63177 Aubière, France

${ }^{2}$ University of Gent, B-9000 Gent, Belgium

${ }^{3}$ Old Dominion University, Norfolk, VA 23529

${ }^{4}$ University of Regina, Regina, SK S4S OA2, Canada

${ }^{5}$ Kent State University, Kent $\mathrm{OH} 44242$

${ }^{6}$ California State University, Los Angeles, CA 90032

${ }^{7}$ College of William and Mary, Williamsburg, VA 23187

${ }^{8}$ CEA Saclay, F-91191 Gif-sur-Yvette, France

${ }^{9}$ Temple University, Philadelphia, PA 19122

${ }^{10}$ University of Georgia, Athens, GA 30602

${ }^{11}$ Massachusetts Institute of Technology, Cambridge, MA 02139

${ }^{12}$ Institut de Physique Nucléaire, F-91406 Orsay, France

${ }^{13}$ Florida International University, Miami, FL 33199

${ }^{14}$ University of Maryland, College Park, MD 20742

${ }^{15}$ University of New Hampshire, Durham, NH 03824

${ }^{16}$ Thomas Jefferson National Accelerator Facility, Newport News, VA 23606

${ }^{17}$ INFN, Sezione Sanità and Istituto Superiore di Sanità, 00161 Rome, Italy

${ }^{18}$ University of Kentucky, Lexington, KY 40506

${ }^{19}$ INFN, Sezione di Bari and University of Bari, 70126 Bari, Italy

${ }^{20}$ Laboratoire de Physique Subatomique et de Cosmologie, F-38026 Grenoble, France

${ }^{21}$ Duke University, Durham, NC 27706

${ }^{22}$ Hampton University, Hampton, VA 23668

${ }^{23}$ Rutgers, The State University of New Jersey, Piscataway, NJ 08855

${ }^{24}$ Kharkov Institute of Physics and Technology, Kharkov 61108, Ukraine

${ }^{25}$ Syracuse University, Syracuse, NY 13244

${ }^{26}$ Yamagata University, Yamagata 990, Japan

${ }^{27}$ Yerevan Physics Institute, Yerevan 375036, Armenia

${ }^{28}$ Tohoku University, Sendai 980, Japan

${ }^{29}$ Princeton University, Princeton, NJ 08544

${ }^{30}$ INFN, Sezione di Lecce, 73100 Lecce, Italy

${ }^{31}$ University of Virginia, Charlottesville, VA 22901

${ }^{32}$ State University of New York at Stony Brook, Stony Brook, NY 11794 


\author{
${ }^{33}$ Norfolk State University, Norfolk, VA 23504 \\ ${ }^{34}$ Florida State University, Tallahassee, FL 32306 \\ ${ }^{35}$ North Carolina Central University, Durham, NC 27707 \\ ${ }^{36}$ Harvard University, Cambridge, MA 02138
}

\begin{abstract}
Virtual Compton Scattering is studied at the Thomas Jefferson National Accelerator Facility at low Center-of-Mass energies, below pion threshold. Following the Low Energy Theorem for the $e p \rightarrow e p \gamma$ process, we obtain values for the two structure functions $P_{L L}-P_{T T} / \epsilon$ and $P_{L T}$ at four-momentum transfer squared $\mathrm{Q}^{2}=0.92$ and $1.76 \mathrm{GeV}^{2}$.

PACS numbers: 13.60.-r,13.60.Fz
\end{abstract}

The electric and magnetic polarizabilities of the nucleon reflect its response to a static electromagnetic field. These are fundamental observables of the ground state, closely related to the entire excitation spectrum of the nucleon. The polarizabilities of the proton have been measured in Real Compton Scattering (RCS) experiments $\gamma p \rightarrow \gamma p$; see e.g. 1]. Contrary to atomic polarizabilities, which are of the size of the atomic volume [2], the proton electric polarizability is much smaller than one cubic fm, the volume scale of a nucleon. In a simplified harmonic oscillator model, such a small electric polarizability is a natural indication of the intrinsic relativistic character of the nucleon. The smallness of the proton magnetic polarizability $\beta_{M}$ relative to $\alpha_{E}$ reflects a strong cancellation of para- and dia-magnetism in the proton.

A theoretical study of the Virtual Compton Scattering (VCS) reaction $\gamma^{*} p \rightarrow \gamma p$, at threshold but at arbitrary virtuality $Q^{2}$ of the initial photon, led to the concept of Generalized Polarizabilities (GP) [3]. The GPs measure the spatial variation of the polarization of the proton, induced by external electric- or magnetic-dipole fields as a function of $Q^{2}$. They provide an original way to probe the nucleon structure. After the NE-18 experiment [4] and the pioneering VCS experiment at MAMI [5], the E93-050 experiment [6] was performed at higher energies at Jefferson Lab (JLab). The studied reaction channel is the exclusive photon electroproduction $e p \rightarrow e p \gamma$. In this Letter, we report the results of the low-energy expansion (LEX) analysis of this experiment, based on the deviations of the cross section with respect to the Low Energy Theorem. In a companion Letter (referred to as II) we report the results of a Dispersion Relation analysis of our data [7].

According to P. Guichon et al. [3], the unpolarized cross section for the reaction $e p \rightarrow e p \gamma$ at small momentum $q^{\prime}$ of the final photon can be written:

$$
\begin{array}{r}
d^{5} \sigma^{E X P}=d^{5} \sigma^{B H+B o r n}+q^{\prime} \phi \Psi_{0}+\mathcal{O}\left(q^{2}\right), \\
\Psi_{0}=v_{1}\left(P_{L L}-\frac{1}{\epsilon} P_{T T}\right)+v_{2} P_{L T}
\end{array}
$$

where $\phi, v_{1}, v_{2}$ are kinematical coefficients, $q^{\prime}$ is the modulus of the three-momentum of the final photon in the $(\gamma p) \mathrm{CM}$ frame and $\epsilon$ is the virtual photon polarization. The differential elements of $d^{5} \sigma$ are the scattered electron momentum and solid angle in the lab frame and the proton solid angle in the $(\gamma p) \mathrm{CM}$ frame. $\Psi_{0}$ contains the effect of the GPs through the structure functions $P_{L L}$,
$P_{T T}, P_{L T} . \Psi_{0}$ represents the effect of GPs to first order in $q^{\prime}$, or equivalently the truncation to electric- or magnetic-dipole radiation. It has been shown to contain six independent (dipole) GPs [8, 9] , combined into the structure functions. $d^{5} \sigma^{B H+B o r n}$ corresponds to the coherent sum of the Bethe-Heitler (BH) and the VCS Born amplitudes. It depends only on the elastic form factors of the proton and is a particular case of Low's low-energy theorem 10] for threshold photon production.

The goal of the measurement, performed at fixed $\epsilon$, is to extract the two structure functions $P_{L L}-P_{T T} / \epsilon$ and $P_{L T}$ by extrapolating the quantity

$$
\left(d^{5} \sigma^{E X P}-d^{5} \sigma^{B H+B o r n}\right) /\left(q^{\prime} \phi\right)
$$

to $q^{\prime}=0$. This is done at a fixed value of $q$, the $\mathrm{CM}$ three-momentum of the VCS virtual photon. Thus, for the small but non-vanishing values of $q^{\prime}$ that we consider, the corresponding squared momentum $Q^{2}=q^{2}-q_{0}^{2}$ depends on $q^{\prime}$ (through the CM virtual photon energy $q_{0}$ ). Though this dependence is very weak for our kinematical conditions, we point out that in the following, $Q^{2}$ will refer to the value corresponding to $q^{\prime}=0$, that is $Q^{2}=2 M_{p} \cdot\left(\sqrt{M_{p}^{2}+q^{2}}-M_{p}\right)$. Without listing the complete expression of the structure functions in terms of the GPs [5, 11], we just mention that $P_{L L}$ is proportional to the electric GP $\alpha_{E}\left(Q^{2}\right)$ and that $P_{L T}$ contains the magnetic GP $\beta_{M}\left(Q^{2}\right)$. When $Q^{2}$ tends to zero, these two GPs give the well-known electric and magnetic polarizabilities obtained in RCS. The structure function $P_{T T}$ is a combination of spin-flip GPs.

The apparatus and running conditions of the JLab experiment are detailed elsewhere 12, 13, 14, 15, 16, 17. Here a brief summary is given. An electron beam of $4.030 \mathrm{GeV}$ energy was directed onto a $15 \mathrm{~cm}$ liquid hydrogen target. The two Hall A Spectrometers were used to detect the scattered electron and the outgoing proton in coincidence, allowing the identification of the exclusive reaction $e p \rightarrow e p \gamma$ by the missing-mass technique. This experiment makes use of the full capabilities of the accelerator and the Hall A instrumentation [18]: 100\% duty cycle, high resolution spectrometers, high luminosities (up to $4 \times 10^{38} \mathrm{~cm}^{-2} \cdot \mathrm{s}^{-1}$ ).

The data for the LEX analysis are divided into two subsets corresponding to data taking in two different ranges of $Q^{2}:[0.85,1.15]$ and $[1.6,2.1] \mathrm{GeV}^{2}$. Spectrometer optics are optimized for the experiment, and dedicated 
procedures allow adjustment of the main experimental offsets in energies, angles and positions. For each event, variables such as $q^{\prime}$, or the CM polar and azimuthal angles $\theta$ and $\varphi$ of the outgoing photon w.r.t. $\vec{q}$, are obtained by reconstructing the missing particle. The acceptance calculation is provided by a dedicated Monte-Carlo simulation [19] including resolution effects. Radiative corrections are applied following the exponentiation method of Ref. [20], the acceptance-dependent part being implemented in the simulation. The acceptance calculation requires a realistic shape for the sampling cross section. For this reason, simulated events are generated using the (BH+Born) cross section plus a first-order GP effect, introduced iteratively. Clean event samples are obtained by applying the event selection method. It involves simple cuts, e.g. a window around 0 in missing mass squared to select the photon peak, or the condition $q^{\prime}<126 \mathrm{MeV} / \mathrm{c}$ to stay below pion threshold. Other cuts are less trivial; special attention was paid to obtain a well-defined acceptance and to eliminate protons punching through the spectrometer entrance collimator. The same cuts are applied to experimental and simulated events. The absolute normalization of the cross section is obtained from the knowledge of the beam charge, target density, and detectors efficiencies. More details can be found in Refs. 13, 14, 15.

For each of the two data sets, the photon electroproduction cross section is determined at a fixed value of $q$ (1.080 and $1.625 \mathrm{GeV} / \mathrm{c})$ and $\epsilon(0.95$ and 0.88). Events are binned in $q^{\prime}(30 \mathrm{MeV} / \mathrm{c}$ wide), and in $\theta$ and $\varphi$ (see Fig. (1). The advantage of the JLab experiment is that it produces a large out-of-plane acceptance, thanks to the Lorentz boost (CM $\rightarrow$ lab) focusing the outgoing proton around $\vec{q}$. An example of out-of-plane cross-section data is given in Fig. 1. At the lowest $q^{\prime}$ of $45 \mathrm{MeV} / \mathrm{c}$, the GP effect is very small $\left(<3 \%\right.$ of $\left.d^{5} \sigma\right)$ and not visible on the plot; at the highest $q^{\prime}$ below pion threshold $(105 \mathrm{MeV} / \mathrm{c})$ it reaches $10-15 \%$. We confirmed the central value of the absolute normalization of the experiment to the $1 \%$ level in the lowest $q^{\prime}$-bin, where the experimental cross section is close to the known (BH+Born) cross section. For this test and throughout our analyses, the $(\mathrm{BH}+\mathrm{Born})$ cross section is calculated using the parametrization of Ref. 21] for the proton EM form factors.

The method to extract the structure functions is deduced from Eq. 1 For each bin in $(\theta, \varphi)$, one measures $d^{5} \sigma^{E X P}$ at several finite values of $q^{\prime}$, and extrapolates the quantity $\Delta \mathcal{M}=\left(d^{5} \sigma^{E X P}-d^{5} \sigma^{B H+B o r n}\right) /\left(\phi q^{\prime}\right)$ to $q^{\prime}=0$, yielding the value of $\Psi_{0}$. In our data, $\Delta \mathcal{M}$ does not exhibit any significant $q^{\prime}$-dependence, so the extrapolation to $q^{\prime}=0$ is done in each bin in $(\theta, \varphi)$ by averaging $\Delta \mathcal{M}$ over $q^{\prime}$. The resulting $\Psi_{0}$ term is then fitted as a linear combination of two free parameters, which are the structure functions $P_{L L}-P_{T T} / \epsilon$ and $P_{L T}$ (see Fig. 2). Their statistical error is given by the fit, and their systematic error is calculated from four sources added quadratically: 1) $\pm 2 \mathrm{MeV}$ on beam energy, 2) $\pm 0.5 \mathrm{mrad}$ on horizontal angles, 3$) \pm 2.3 \%$ on overall absolute cross
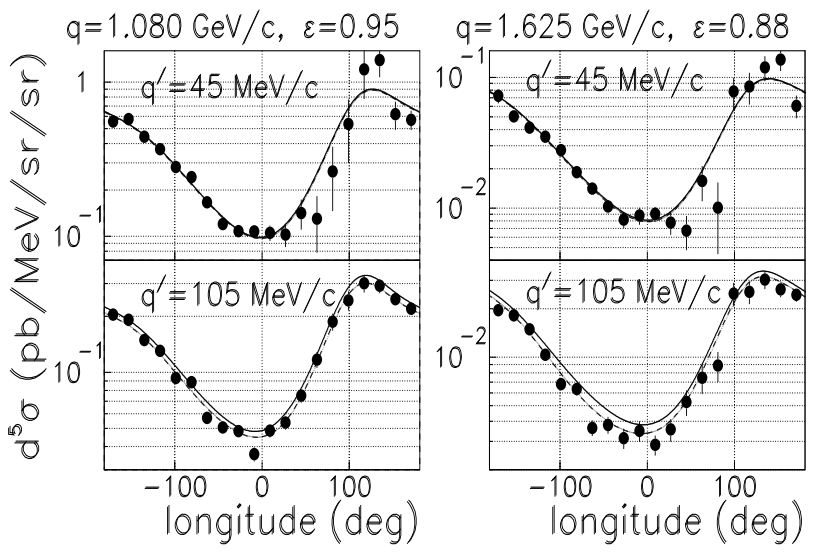

FIG. 1: $(e p \rightarrow e p \gamma)$ cross section for the lowest and highest $q^{\prime}$ bin, at $40^{\circ}$ out-of-plane (including symmetry w.r.t. the lepton plane). Only statistical errors are shown. Here the out-of-plane angle (or latitude) is the polar angle of the outgoing photon when the polar axis is chosen perpendicular to the lepton plane. The abscissa is the azimuthal angle (or longitude) using this convention; negative values are defined as in Ref. [5]. The full curve is the (BH+Born) cross section, the dashed curve includes the first-order GP effect (Eq. 1) as fitted in this analysis.

section normalization and 4) $\pm 2 \%$ due to possible cross section shape distortions. The value of the reduced $\chi^{2}$ of the fit (1.22 and 1.50, cf. Fig. 2) is one way to express that the LEX holds reasonably well at our kinematics.

Our results, presented in Table \ together with the previous results at lower $Q^{2}$, show a strong fall-off of the structure functions with momentum transfer, similarly to form factors. This behavior is expected since GPs can be seen as "form factors of a nucleon embedded in an EM field". From a comparison with theoretical predictions, one should point out that most model calculations of the

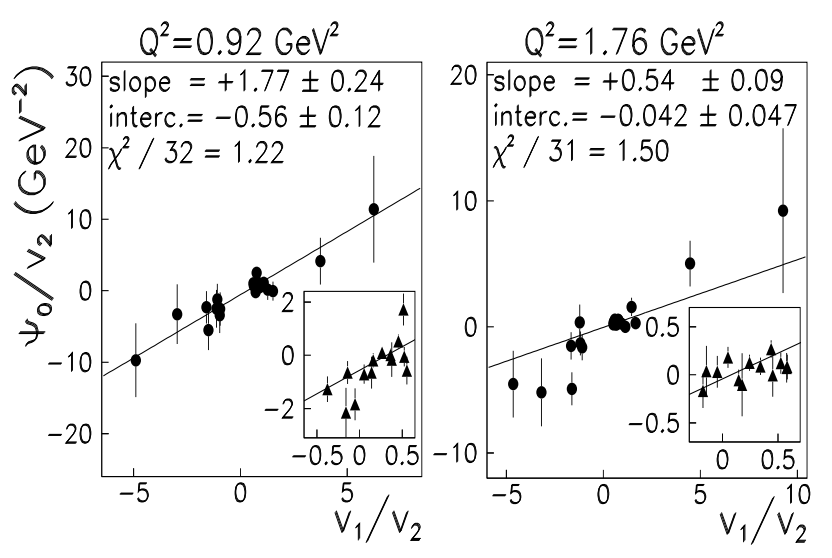

FIG. 2: A graphical representation of the LEX fit (straight line) for each data set. Black circles correspond to out-ofplane data, and the inner plot is a zoom on the lepton plane data (triangles). $\Psi_{0}, v_{1}$ and $v_{2}$ are defined in the text. 
TABLE I: Compilation of the VCS structure functions. The first line corresponds to the RCS result [1]. In all cases the first error is statistical, and the second one is the total systematic error.

\begin{tabular}{ccccc}
\hline \hline Ref. & $\begin{array}{c}Q^{2} \\
\left(\mathrm{GeV}^{2}\right)\end{array}$ & $\epsilon$ & $\begin{array}{c}P_{L L}-P_{T T} / \epsilon \\
\left(\mathrm{GeV}^{-2}\right)\end{array}$ & $\begin{array}{c}P_{L T} \\
\left(\mathrm{GeV}^{-2}\right)\end{array}$ \\
\hline \multicolumn{4}{c}{ Previous experiments } \\
\hline$[1]$ & 0 & & 81.3 & -5.4 \\
& & & $\pm .0 \pm 3.4$ & $\pm 1.3 \pm 1.9$ \\
{$[5]$} & 0.33 & 0.62 & 23.7 & -5.0 \\
& & & $\pm 2.2 \pm 4.3$ & $\pm 0.8 \pm 1.8$ \\
\hline & \multicolumn{4}{c}{ This experiment } \\
\hline & 0.92 & 0.95 & $\mathbf{1 . 7 7}$ & $\mathbf{- 0 . 5 6}$ \\
& \multirow{4}{*}{1.76} & 0.88 & $\mathbf{0 . 5 4}$ & $\mathbf{- 0 . 0 4 2}$ \\
& & $\pm 0.09 \pm 0.20$ & $\pm 0.047 \pm 0.055$ \\
\hline \hline
\end{tabular}

GPs, e.g. Refs. 22, 23, 24] are valid at much smaller $Q^{2}$ than the ones covered in this experiment. One notable exception is the Dispersion Relation (DR) model [25]; our extraction of VCS structure functions in the same experiment, based on the DR approach, is the subject of a companion Letter [7]. The results presented here should stimulate theoretical calculations of GPs at intermediate and high $Q^{2}$. It would be of great interest if the models that predict the form factors $G_{E}$ and $G_{M}$ at $Q^{2} \geq 1$ $\mathrm{GeV}^{2}$ could give predictions of the GPs in the same $Q^{2}$ range.

In summary, we have studied the process $e p \rightarrow e p \gamma$ using the unique capabilities of the JLab electron beam and the Hall A instrumentation. We have measured the two unpolarized structure functions of Virtual Compton Scattering $P_{L L}-P_{T T} / \epsilon$ and $P_{L T}$, providing a new piece of information on the electromagnetic structure of the nucleon, namely on the behavior of the proton Generalized Polarizabilities in the range $Q^{2} \sim 1-2 \mathrm{GeV}^{2}$. Other VCS experiments at low energy will measure these structure functions (MIT-Bates 26], MAMI 27]) and double polarization is foreseen [27] as a way to separate the six lowest order GPs.

We thank the JLab accelerator staff and the Hall A technical staff for their dedication. This work was supported by DOE contract DE-AC05-84ER40150 under which the Southeastern Universities Research Association (SURA) operates the Thomas Jefferson National Accelerator Facility. We acknowledge additional grants from the US DOE and NSF, the French Centre National de la Recherche Scientifique and Commissariat à l'Energie Atomique, the Conseil Régional d'Auvergne, the FWO-Flanders (Belgium) and the BOF-Gent University. We thank M.Vanderhaeghen and B.Pasquini for fruitful discussions and the organization of VCS workshops at ECT* (Trento).
[1] V. Olmos de Leon et al., Eur. Phys. J. A10, 207 (2001).

[2] V. A. Dzuba, V. V. Flambaum, and O. P. Sushkov (1997), hep-ph/9709251.

[3] P. A. M. Guichon, G. Q. Liu, and A. W. Thomas, Nucl. Phys. A591, 606 (1995).

[4] J. F. J. van den Brand et al., Phys. Rev. D52, 4868 (1995).

[5] J. Roche et al., Phys. Rev. Lett. 85, 708 (2000).

[6] P. Y. Bertin, C. Hyde-Wright, P. A. M. Guichon, et al. (1993), experiment E93-050, URL http://hallaweb.jlab.org/experiment/E93-050/vcs.html

[7] G. Laveissière et al., Letter II, to be submitted to Phys. Rev. Lett.

[8] D. Drechsel, G. Knochlein, A. Metz, and S. Scherer, Phys. Rev. C55, 424 (1997).

[9] D. Drechsel, G. Knochlein, A. Y. Korchin, A. Metz, and S. Scherer, Phys. Rev. C57, 941 (1998).

[10] F. E. Low, Phys. Rev. 110, 974 (1958).

[11] P. A. M. Guichon and M. Vanderhaeghen, Prog. Part. Nucl. Phys. 41, 125 (1998).

[12] G. Laveissière et al. (2003), to be submitted to Phys. Rev. C, nucl-ex/0308009.

[13] N. Degrande, Ph.D. thesis, Gent University (2001).

[14] S. Jaminion, Ph.D. thesis, Université Blaise Pascal (2001), DU 1259.
[15] C. Jutier, Ph.D. thesis, Old Dominion University and Université Blaise Pascal (2001), DU 1298.

[16] G. Laveissière, Ph.D. thesis, Université Blaise Pascal (2001), DU 1309.

[17] L. Todor, Ph.D. thesis, Old Dominion University (2000).

[18] J. Alcorn et al., accepted by NIM A.

[19] L. Van Hoorebeke et al., to be submitted to NIM A.

[20] M. Vanderhaeghen, J. M. Friedrich, D. Lhuillier, D. Marchand, L. Van Hoorebeke, and J. Van de Wiele, Phys. Rev. C62, 025501 (2000).

21] E. J. Brash, A. Kozlov, S. Li, and G. M. Huber, Phys. Rev. C65, 051001 (2002).

[22] T. R. Hemmert, B. R. Holstein, G. Knochlein, and D. Drechsel, Phys. Rev. D62, 014013 (2000).

[23] C. W. Kao and M. Vanderhaeghen, Phys. Rev. Lett. 89, 272002 (2002).

[24] B. Pasquini, S. Scherer, and D. Drechsel, Phys. Rev. C63, 025205 (2001).

[25] D. Drechsel, B. Pasquini, and M. Vanderhaeghen, Phys. Rept. 378, 99 (2003).

[26] J. Shaw, R. Miskimen, et al. (1997), MIT-Bates proposal E97-03.

[27] H. Merkel and N. d'Hose (2000), spokspersons, MAMI Proposal. 